Comment. Math. Helv. 74 (1999) 615-641

(C) 1999 Birkhäuser Verlag, Basel

$0010-2571 / 99 / 040615-27 \$ 1.50+0.20 / 0$

Commentarii Mathematici Helvetici

\title{
Mutation and gauge theory I: Yang-Mills invariants
}

\author{
Daniel Ruberman
}

\begin{abstract}
Mutation of 3-manifolds (cutting and regluing along a genus 2 surface using a central involution) is shown to preserve the instanton Floer homology of homology 3-spheres. A related operation on 4-manifolds is shown to preserve the Donaldson polynomial invariant.
\end{abstract}

Mathematics Subject Classification (1991). 57A57, 58G10.

Keywords. Floer homology, mutation, Donaldson invariants.

Mutation is an operation on 3-manifolds containing an embedded surface $\Sigma$ of genus 2. It is defined using the unique involution, called $\tau$, of $\Sigma$ with the property that $\Sigma / \tau \cong S^{2}$. In brief, given a 3 -manifold $M$ containing $\Sigma$, its mutant $M^{\tau}$ is obtained by cutting $M$ along $\Sigma$, and regluing using $\tau$. This operation was introduced in [40] as the analogue for closed manifolds of the mutation operation on knots described by Conway [12]. It is not easy to distinguish a 3-manifold from its mutant - there is a long list of invariants which they have in common: their Gromov norm [40], Reidemeister torsion [37], Chern-Simons and $\eta$-invariants $[32,31]$ (if $M$ is hyperbolic), Casson's invariant [28], and many of the 'quantum' invariants of 3-manifolds [23, 24, 30, 38, 39].

In this article, we will show that the instanton Floer homology [18] and Zgraded instanton homology [17] of homology spheres are unchanged by mutation.

Theorem 1. Let $M$ be an oriented homology 3-sphere, with (instanton) Floer homology $H F_{*}(M)$, which contains a genus-2 surface, and let $M^{\tau}$ be the result of mutation along $\Sigma$. Then $H F_{*}(M) \cong H F_{*}\left(M^{\tau}\right)$. Similarly, if $H F^{\mu}$ denotes the Z-graded instanton homology of Fintushel-Stern, then $H F_{*}^{\mu}(M) \cong H F_{*}^{\mu}\left(M^{\tau}\right)$.

In section 2 we will define two types of mutation operations on certain 4manifolds, and show that they preserve the Donaldson invariant. A companion article (in preparation) will show that the 3-dimensional Seiberg-Witten analogue of Casson's invariant is unchanged by mutation.

Theorem 1 provides an alternate proof of P. Kirk's result [28] on the Casson

The author was partially supported by NSF Grant 4-50645. 
invariant. In his paper [25], A. Kawauchi constructs homology spheres with the same Floer homology as their mutants, and remarks that the general case does not seem to be known. On reviewing Kawauchi's paper for Mathematical Reviews, my interest in the problem was stimulated by this remark. The papers of Kirk and Kawauchi are based on the connection between surgery and Casson's invariant (respectively Floer homology), whereas we will proceed directly from the definition of Floer homology in terms of SU(2)-representations. The restriction to homology spheres is largely for technical convenience; it is likely that the proof of theorem 1 would extend to Floer-type theories [5, 8] defined for more general 3-manifolds.

Notation. For the rest of the paper, $M$ will denote a closed, oriented 3-manifold, and $\Sigma$ a genus- 2 surface. For any space $X$, and Lie group $G$, the space of representations of $\pi_{1}(X)$ into $G$ will be denoted $\operatorname{Rep}(X, G)$. The equivalence classes (under the relation of conjugacy by elements of $G$ ) will be denoted $\chi(X, G)$. If it is obvious what group is being discussed, then the ' $G$ ' may be dropped.

\section{Mutation and floer homology}

The involution $\tau$ has several related properties which are responsible for the equality of the invariants cited above after mutation. The basic one is that any simple closed curve $\gamma$ on $\Sigma$ is isotopic to one which is taken to itself by $\tau$, perhaps with a reversal of orientation. This implies that $\tau$ is in the center of the mapping class group, but more importantly for our purposes, implies the following lemma, well-known in certain circles.

Lemma 1.1. Let $\varphi: \pi_{1}(\Sigma) \rightarrow \mathrm{SU}(2)$ be a representation. Then $\varphi \circ \tau_{*}$ is conjugate to $\varphi$. The same is true for representations of $\pi_{1}(\Sigma)$ in $\mathrm{SL}_{2}(\mathbf{R})$.

A similar lemma, concerning instead representations in $\mathrm{SL}_{2}(\mathbf{C})$, may be found in $\S 2$ of [40], and the proof there may be adapted, mutatis mutandis.

Remark. In the field of 'quantum invariants' of 3-manifolds, this lemma is seen as a reflection of a self-duality of certain representations of $\mathrm{SU}(2)$. This duality does not hold for larger rank unitary groups, so one expects that invariants based on, say, $\mathrm{SU}(3)$ representations, would change under mutations. We will return to this point in Section 2.

Using the standard correspondence between representations and flat connections, this lemma says that for any flat $\mathrm{SU}(2)$-connection $\alpha$ on $\Sigma$, the pull-back $\tau^{*} \alpha$ is gauge equivalent to $\alpha$. If $\alpha$ is reducible, then there is a choice of (constant) gauge transformation $g \in \operatorname{Stab}(\alpha) / \mathbf{Z}_{2}$ with $\tau^{*} \alpha=g^{*} \alpha$. Any such $g$ defines an automorphism $\hat{\tau}$ of the connection $\alpha$ on the trivial bundle, as the following 
composition:

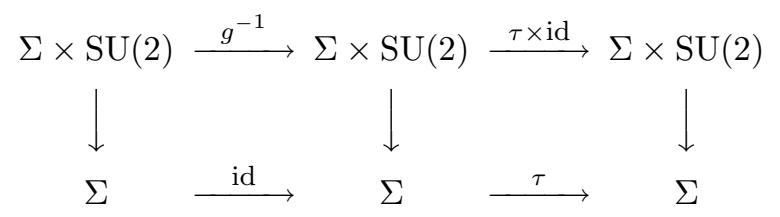

By definition, $\hat{\tau}$ covers $\tau$, and induces an automorphism $\tau^{*}$ of the $s u_{2}$-valued forms $\Omega^{*}(\Sigma ; \operatorname{ad} \alpha)$, and the twisted cohomology groups $H^{*}(\Sigma ; \operatorname{ad} \alpha)$.

Starting from Lemma 1.1, there is an obvious path to take in showing the equality of $\mathrm{HF}_{*}$ of mutant homology spheres. Let $\mathrm{CF}_{*}$ denote the chain complex which computes the instanton homology; in favorable circumstances this has a basis indexed by the flat $\mathrm{SU}(2)$-connections on $M \times \mathrm{SU}(2)$, or equivalently by $\chi(M, \mathrm{SU}(2))$. The $\mathbf{Z} / 8 \mathbf{Z}$-grading is given by spectral flow. For $\mu \in \mathbf{R}$ such that $\mu \neq \operatorname{CS}(\alpha)$ for any $\alpha \in \chi(M)$, Fintushel and Stern [17] have defined Z-graded chain groups $\mathrm{CF}_{*}^{\mu}$. These have the same basis as $\mathrm{CF}_{*}$, but the grading is lifted from $\mathbf{Z} / 8 \mathbf{Z}$ to $\mathbf{Z}$ using the monotonicity properties of the Chern-Simons invariant.

Suppose that $\Sigma \subset M$, separating $M$ into two components whose closures will be denoted $A$ and $B$. When $M$ has a Riemannian metric, the metric will be assumed to be a product in a neighborhood of $\Sigma$. Moreover, we will assume that $\tau$ is an isometry of the restriction of the metric to $\Sigma$. In this notation, $M^{\tau}$ will be given by $A \cup_{\tau} B$, and inherits a metric from $M$. Given a representation $\varphi$ of $\pi_{1}(M)$ in $\mathrm{SU}(2)$, let $\varphi_{\Sigma}$ denote its restriction to $\pi_{1}(\Sigma)$, with $\varphi_{A}$ (resp. $\varphi_{B}$ ) the restrictions to $\pi_{1}(A)$ (resp. $\pi_{1}(B)$.) Choose an element $g \in \mathrm{SU}(2)$ with $\varphi_{\Sigma} \circ \tau_{*}=g^{-1} \varphi_{\Sigma} g$, and conjugate $\varphi_{B}$ by $g$, to get a representation $\varphi^{\tau}$ of $\pi_{1}\left(M^{\tau}\right)$. Thus we get a sort of correspondence between $\chi(M, \mathrm{SU}(2))$ and $\chi\left(M^{\tau}, \mathrm{SU}(2)\right)$, which should lead to an isomorphism on instanton homology.

There are several issues with which one must deal:

(i) The character variety $\chi(M)$ may not consist of a finite number of smooth points.

(ii) If $\varphi_{\Sigma}$ is reducible, then there is a choice (parameterized by $\operatorname{Stab}\left(\varphi_{\Sigma}\right) / \mathbf{Z}_{2}$ ) of elements $g$ conjugating $\varphi_{\Sigma}$ to $\varphi_{\Sigma} \circ \tau_{*}$.

Even if these problems do not arise, so that we have a sensible map $T_{*}: \mathrm{CF}_{*}(M) \rightarrow$ $\mathrm{CF}_{*}\left(M^{\tau}\right)$ (and $T_{*}^{\mu}$ in the $\mathbf{Z}$-graded case) we would need to show:

(iii) The map $T_{*}$ preserves the $\mathbf{Z} / 8 \mathbf{Z}$ grading in $\mathrm{CF}_{*}$ and $T_{*}^{\mu}$ the $\mathbf{Z}$ grading in $\mathrm{CF}_{*}^{\mu}$.

(iv) $T_{*}$ and $T_{*}^{\mu}$ are chain maps.

It turns out that $T_{*}$ is not a chain map, although $T_{*}^{\mu}$ is.

The first two issues will be handled using a perturbation, as the experts will have surmised. The existence of a chain map related to $T_{*}$ is derived from a basic geometric construction, which we present in the next section. 

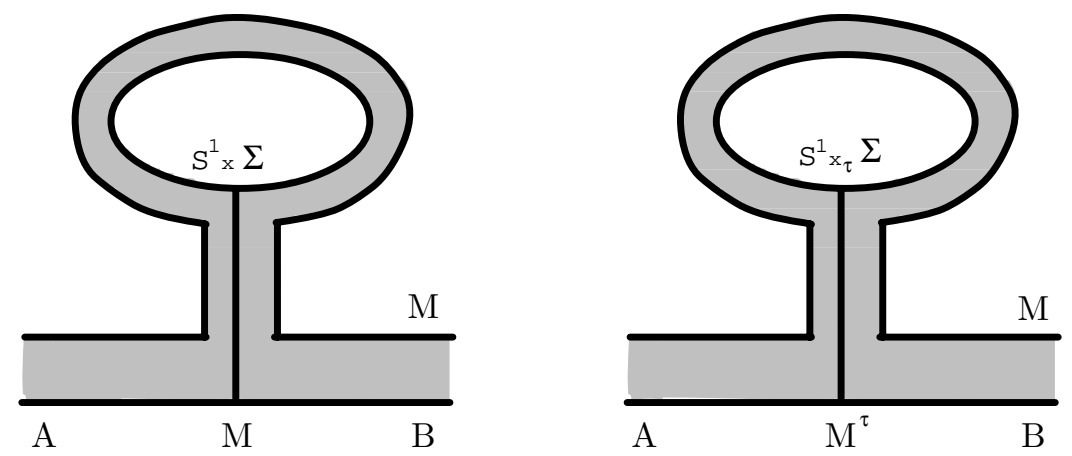

Figure 1.

\subsection{The basic cobordism}

The functoriality of $\mathrm{HF}_{*}$ with respect to oriented cobordisms suggests a method to show that the correspondence $T_{*}$ is a chain map. One would need (among other things) a cobordism between $M$ and $M^{\tau}$, over which a representation $\varphi$ and its cut-and-pasted cousin $\varphi^{\tau}$ would extend. We do not know how to construct such a cobordism, but we can come very close. We use a variation of the idea in $\S 2$ of our earlier paper [31] to construct a cobordism having an additional boundary component, which will be filled in as the boundary of a 4-dimensional orbifold.

Start with a copy of $\Sigma$ sitting inside $M$, so that $M=A \cup_{\Sigma} B$. The labeling of the two components is arbitrary, but having chosen it we can make the following convention: $\Sigma$ is to be oriented as the boundary of $A$ (and hence $-\Sigma=\partial B$.) The mutated manifold $M^{\tau}$ is then formally a quotient space $(A \amalg B) / \sim$, where ' $\sim$ ' identifies $x \in \partial B$ with $\tau(x)$ in $\partial A$. (Since $\tau$ is an involution, it doesn't much matter how we do this, but some care in making the identifications now will help in the calculations later.) Fix a collar neighborhood $I \times \Sigma$ in $M$.

Consider the manifold $W\left(=W\left(M, S^{1} \times \Sigma\right)\right.$ from [31]) constructed as follows:

$$
W=M \times[0,1 / 4] \cup(I \times \Sigma) \times[1 / 4,3 / 4] \cup M \times[3 / 4,1]
$$

Cut and paste $W$ along $\Sigma \times[0,1]$, using the involution $\tau \times \operatorname{id}_{I}$, to obtain a new manifold $W^{\tau}$.

From Figure 1, it is clear that the boundary of $W^{\tau}$ consists of a copy of $M$, a copy of $M^{\tau}$, and a copy of the mapping torus $S^{1} \times_{\tau} \Sigma$. From the point of view of index theory, $S^{1} \times_{\tau} \Sigma$ will be seen to capture the difference between $M$ and $M^{\tau}$.

Lemma 1.2. The mapping torus $S^{1} \times{ }_{\tau} \Sigma$ is a Seifert fibered space $S^{2}(2,2,2,2,2,2 ; e)$ over $S^{2}$, with 6 fibers of multiplicity 2 and Euler class $\delta-3$.

Proof. Let $\pi: \Sigma \rightarrow S^{2}$ be the branched double cover for which $\tau$ is the covering transformation. There are 6 branch points, each of order 2 . Because $\pi \circ \tau=\pi$, the 
projection extends to a map $S^{1} \times_{\tau} \Sigma \rightarrow S^{2}$ which is a fibration away from the fixed points. The inverse image of each fixed point is a circle, which is covered by nearby circles with multiplicity 2 . The Euler class calculation may be done directly, or more easily by observing that the first homology group of $S^{1} \times_{\tau} \Sigma$ is $(\mathbf{Z} / 2 \mathbf{Z})^{4} \oplus \mathbf{Z}$. On the other hand, $H_{1}$ of the Seifert fibered space $S^{2}(2,2,2,2,2,2 ; e)$ is given by $(\mathbf{Z} / 2 \mathbf{Z})^{4} \oplus \mathbf{Z} /(e+3) \mathbf{Z}$.

Following the construction ${ }^{\mathrm{TM}}$ of Fintushel and Stern [15], let $V$ be the mapping cylinder of the projection $S^{1} \times_{\tau} \Sigma \rightarrow S^{2}$, and form an orbifold

$$
Y^{\tau}=W^{\tau} \cup_{S^{1} \times_{\tau} \Sigma} V
$$

By Lemma 1.2, there are 6 singular points in $Y^{\tau}$, each the cone on $\mathbf{R P}^{3}$. It is easily checked that $V$ has the homology of $S^{2}$, and that the (rational) self-intersection of the generator of $H_{2}(V)$ is trivial. We will also use the notations $V_{0}$ and $Y_{0}^{\tau}$ for the complement of an open neighborhood of the six singular points.

We will also need the homology and cohomology groups of $W^{\tau}$.

Lemma 1.3. The homology and cohomology groups of $W^{\tau}$ are as follows:

$$
H_{1}=H^{1}=\mathbf{Z}, \quad H_{2}=H^{2}=\mathbf{Z}^{4}, \quad H_{3}=H^{3}=\mathbf{Z}^{2} .
$$

A choice of basis for $H_{1}(\Sigma)$ gives a basis for $H_{2}\left(W^{\tau}\right)$. With respect to a symplectic basis consisting of elements from $\operatorname{ker} H_{1}(\Sigma) \rightarrow H_{1}(A)$ or $H_{1}(B)$, the intersection form is

$$
\left(\begin{array}{ll}
0 & 1 \\
1 & 0
\end{array}\right) \oplus\left(\begin{array}{ll}
0 & 1 \\
1 & 0
\end{array}\right)
$$

Proof. This all follows from the Mayer-Vietoris sequence, using the fact that $\tau_{*}=-1$ on $H_{1}(\Sigma)$. The calculation of the intersection form follows [31, Proposition $2.1]$.

\subsection{Extending representations}

Until further notice, all representations will be in the Lie group $\mathrm{G}=\mathrm{SU}(2)$ or $\mathrm{SO}(3)$, so that, for example, $\chi\left(\pi_{1}(M)\right)$ refers to the $\mathrm{SU}(2)$-character variety of $M$. (Since $M$ is a homology sphere, the varieties of $\mathrm{SU}(2)$ and $\mathrm{SO}(3)$ representations are the same.) In this section, we will show that every $\mathrm{SU}(2)$-representation of $\pi_{1}(M)$ extends to $\pi_{1}\left(W^{\tau}\right)$ and then to an $\mathrm{SO}(3)$ representation of $\pi_{1}^{\text {orb }}\left(Y^{\tau}\right)$. There always at least two $\mathrm{SU}(2)$ extensions over $\pi_{1}\left(W^{\tau}\right)$, but upon passage to $\mathrm{SO}(3)$ there is only one, provided that the representation is irreducible when restricted to $\Sigma$. By restricting to $\pi_{1}\left(M^{\tau}\right)$ we get the one-to-one correspondence between $\operatorname{Rep}(M)$ and $\operatorname{Rep}\left(M^{\tau}\right)$ referred to above. It is not obvious (and we will not need to know) that 


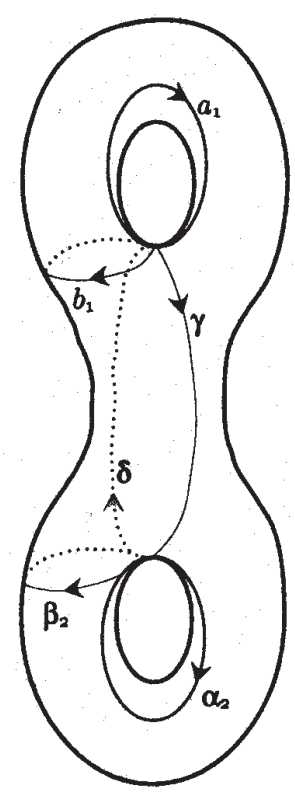

Figure 2 .

this is continuous; it is perhaps better to view the maps $\operatorname{Rep}(M) \leftarrow \operatorname{Rep}\left(W^{\tau}\right) \rightarrow$ $\operatorname{Rep}\left(M^{\tau}\right)$ as defining a correspondence in the sense of algebraic geometry.

There is a standard correspondence between $\mathrm{SU}(2)$ representations and flat $\mathrm{SU}(2)$ connections, with conjugacy of representations going over to gauge equivalence. We will use the two notions interchangeably, without varying the notation. In this interpretation, the connection $\alpha^{\tau}$ is identical to $\alpha$ on $A$, but differs from $\alpha$ on $B$ by a constant gauge transformation.

Regard $\pi_{1}(M)$ as being presented as an amalgamation as follows:

$$
\pi_{1}(M)=\left\langle\pi_{1}(A), \pi_{1}(B) \mid i_{a}(g)=i_{b}(g) \forall g \in \pi_{1}(\Sigma)\right\rangle
$$

Here $i_{a}, i_{b}$ are the maps induced by the inclusions of $\Sigma$ into the two sides. In this notation, the fundamental group of $W^{\tau}$ is easily calculated, using van Kampen's theorem.

$$
\pi_{1}\left(W^{\tau}\right)=\left\langle\pi_{1}(A), \pi_{1}(B), z \mid z^{-1} i_{a}(g) z=i_{b}\left(\tau_{*}(g)\right) \forall g \in \pi_{1}(\Sigma)\right\rangle
$$

The fundamental group of $M^{\tau}$ has a similar description.

We will need a more explicit calculation of the effect of $\tau_{*}$ on $\pi_{1}(\Sigma)$. In figure 2 below, the generators of the fundamental group are $a_{1}, b_{1}$ as drawn in the top half of the surface, and $a_{2}, b_{2}$ which are given by $\gamma \alpha_{2} \bar{\gamma}$ and $\gamma \beta_{2} \bar{\gamma}$ respectively. Here, and in what follows, $\bar{x}$ will be used as a synonym for $x^{-1}$. 
Referring to Figure 2, and the curves as labeled therein, we have that

$$
\pi_{1}(\Sigma)=\left\langle a_{1}, b_{1}, a_{2}, b_{2} \mid a_{1} b_{1} \bar{a}_{1} \bar{b}_{1} b_{2} a_{2} \bar{b}_{2} \bar{a}_{2}=1\right\rangle
$$

With respect to these generators, the action of $\tau$ is given by

$$
\begin{aligned}
\tau_{*}\left(a_{1}\right) & =\bar{a}_{1} \\
\tau_{*}\left(b_{1}\right) & =a_{1} \bar{b}_{1} \bar{a}_{1} \\
\tau_{*}\left(a_{2}\right) & =\bar{b}_{1} b_{2} \bar{a}_{2} \bar{b}_{2} b_{1} \\
\tau_{*}\left(b_{2}\right) & =\bar{b}_{1} b_{2} a_{2} \bar{b}_{2} \bar{a}_{2} \bar{b}_{2} b_{1}
\end{aligned}
$$

For any $\mathrm{SU}(2)$-representation $\varphi$, let $\operatorname{Stab}(\varphi)=\mathbf{Z}_{2}, S^{1}$ or $\mathrm{SU}(2)$ be its stabilizer. Lemma 1.1, together with the presentation (2) of $\pi_{1}\left(W^{\tau}\right)$, have the following consequence.

Theorem 1.4. Any $\mathrm{SU}(2)$ representation $\varphi$ of $\pi_{1}(M)$ extends to a representation of $\pi_{1}\left(W^{\tau}\right)$. The set of extensions, up to conjugacy, is in one-to-one correspondence with $\operatorname{Stab}\left(\varphi_{\Sigma}\right)$.

Corollary 1.5. If $\varphi$ is an $\mathrm{SO}(3)$-representation of $\pi_{1}(M)$ whose restriction to $\Sigma$ is irreducible, then it has a unique extension to $\pi_{1}\left(W^{\tau}\right)$.

Proof. Since $M$ is a homology sphere, $\varphi$ has a unique lift to an $\mathrm{SU}(2)$ representation, which has two extensions to $\pi_{1}\left(W^{\tau}\right)$ according to the theorem. But these become the same when projected back to $\mathrm{SO}(3)$.

When $S^{1} \times{ }_{\tau} \Sigma$ is filled in to make the orbifold $Y^{\tau}$, it is no longer the case that flat connections extend, because of the possible holonomy around the $S^{1}$ fiber. However, they do extend as flat orbifold connections.

Theorem 1.6. Let $\varphi$ be an $\mathrm{SO}(3)$-representation of $\pi_{1}(M)$ whose restriction to $\Sigma$ is irreducible. Then it has a unique extension to

$$
\varphi_{Y}: \pi_{1}^{\text {orb }}\left(Y^{\tau}\right)=\pi_{1}\left(Y_{0}^{\tau}\right) \rightarrow \mathrm{SO}(3)
$$

Furthermore, this representation is non-trivial at each singular point in the orbifold, and has $w_{2}\left(\varphi_{Y}\right)$ characterized as follows: it is the unique class in $H^{2}\left(Y_{0}^{\tau} ; \mathbf{Z}_{2}\right)$ with trivial restriction to $S^{1} \times_{\tau} \Sigma$ and with non-trivial restriction to each $\mathbf{R P}^{3}$ component of $\partial Y_{0}^{\tau}$.

Proof. Because $\tau^{2}$ is the identity, the presentation (2) implies that under the extension of $\varphi$ to $\pi_{1}\left(W^{\tau}\right)$, the element $z$ goes to an element of order two in $\mathrm{SO}(3)$. (The hypothesis on $\varphi_{\Sigma}$ implies that $z$ can't go to the identity.) Assuming that 
the base point was chosen to be one of the fixed points of $\tau$ on $\Sigma$, the generator of the local fundamental group near the corresponding singular point of $Y^{\tau}$ is $z$. Hence the representation is non-trivial at that point. The generators for the local fundamental groups of the other singular points are all conjugate to $z$, so the representation is non-trivial at each of these points. That this specifies $w_{2}\left(\varphi_{Y}\right)$, as described in the statement of the theorem, may be proved by examining the long exact sequence of the pair $\left(V_{0}, \partial V_{0}\right)$.

In section 1.4, we will compare the Floer-grading of perturbed flat connections on $M$ and $M^{\tau}$, using the theory of $[9,10,11]$. For connections which are actually flat, there is a more elementary approach, based on the work of Atiyah-PatodiSinger [2, 3] as applied to the operator linearizing the ASD Yang-Mills equations on $W^{\tau}$. This approach requires some information about the $\mathrm{SU}(2)$ representations of $\pi_{1}\left(S^{1} \times_{\tau} \Sigma\right)$, and their associated $\rho$ and Chern-Simons invariants, which we develop in the remainder of this section. This material may safely be skipped by those readers who would prefer to pass directly to the proof of the main theorems on mutation.

Lemma 1.7. The space $\chi\left(S^{1} \times_{\tau} \Sigma\right)$ of $\mathrm{SU}(2)$ representations of $\pi_{1}\left(S^{1} \times_{\tau} \Sigma\right)$ is connected.

In the proof we will consider elements of $\mathrm{SU}(2)$ as unit quaternions.

Proof. The standard presentation of $\pi_{1}\left(S^{1} \times_{\tau} \Sigma\right)$ as an HNN extension is

$$
\pi_{1}\left(S^{1} \times_{\tau} \Sigma\right)=\left\langle\pi_{1}(\Sigma), z \mid z^{-1} g z=\tau_{*}(g) \forall g \in \pi_{1}(\Sigma)\right\rangle
$$

Thus, a representation $\alpha$ of $\pi_{1}\left(S^{1} \times_{\tau} \Sigma\right)$ is determined by its restriction $\alpha_{\Sigma}$ to $\pi_{1}(\Sigma)$, and the choice of the element $z$ conjugating $\alpha_{\Sigma}$ to $\tau^{*}\left(\alpha_{\Sigma}\right)$. It is not hard to show that $z$ is determined up to multiplication by elements of the stabilizer of $\alpha_{\Sigma}$.

The restriction map from $\alpha \rightarrow \alpha_{\Sigma}$ is then generically two-to-one, with connected fibers over the reducible connections on $\Sigma$. We will show that the two preimages of an irreducible connection on $\Sigma$ can be connected via a path in $\chi\left(S^{1} \times_{\tau} \Sigma\right)$. The map to $\chi^{*}(\Sigma)$ is in fact a covering space, so the lemma will follow from the fact that both $\chi^{*}(\Sigma)$ and the full SU(2)-representation variety $\chi(\Sigma)$ are connected. (In fact, $\chi(\Sigma)$ is homeomorphic to $\mathbf{C P}^{3}$, and the reducibles are a singular quartic.)

It suffices to find a path connecting the two preimages (say $\alpha_{1}, \alpha_{1}^{\prime}$ ) of a single irreducible connection. The path will be made of 3 pieces: the first connecting $\alpha_{1}$ to a reducible connection $\alpha_{0}$, the second a path in the reducible stratum from $\alpha_{0}$ to $\alpha_{0}^{\prime}$, and a third connecting $\alpha_{0}^{\prime}$ to $\alpha_{1}^{\prime}$.

Referring to the generators given in Figure 2, let the representation $\alpha_{r}, r \in$ 
$[0,1]$ be given by

$$
\begin{aligned}
\alpha_{r}\left(a_{1}\right) & =\exp \left(-\frac{\pi r}{2} \imath\right) & & \alpha_{r}\left(a_{2}\right)=\exp \left(-\frac{\pi r}{2} \imath\right) \\
\alpha_{r}\left(b_{1}\right) & =\jmath & & \alpha_{r}\left(b_{2}\right)=\jmath \\
\alpha_{r}(t) & =\exp \left(\frac{\pi(1-r)}{2} \jmath\right) & &
\end{aligned}
$$

The representation $\alpha_{r}$ restricts to an irreducible representation on $\Sigma$, for $1 \geq$ $r>0$. Note that for these values of $r$, the other representation with the same restriction to $\Sigma$ differs only in having the opposite sign for $\alpha_{r}(z)$. On the other hand, $\alpha_{0}$ has $\alpha_{0}(z)=\imath \jmath=k$, and is reducible on $\pi_{1}(\Sigma)$, with image lying in the circle subgroup containing $k$. Consider the path $\alpha_{0, s}, s \in[0,1]$ of representations with the same effect on $\pi_{1}(\Sigma)$, but with $\alpha_{0, s}(z)=\imath(\sin (\pi s)+\cos (\pi s) \jmath)$. Since $(\sin (\pi s)+\cos \pi s) \jmath)$ is in the centralizer of $\alpha_{0}\left(\pi_{1}(\Sigma)\right)$, this gives a path from $\alpha_{0}$ to $\alpha_{0}^{\prime}$, which is the same as $\alpha_{0}$ except that $z$ is sent to $-k$. The path $\alpha_{r}^{\prime}$, which is the same as $\alpha_{r}$ except for reversing the sign of $\alpha_{r}(z)$, provides the third piece of the path.

It follows that the variety $\chi\left(S^{1} \times_{\tau} \Sigma\right)$ is has singularities sitting over the reducibles in $\chi(\Sigma)$, but that the part sitting over the irreducible part of $\chi(\Sigma)$ is smooth.

Because the Chern-Simons invariant doesn't change on paths of flat connections, we immediately obtain:

Corollary 1.8. For any $\alpha \in \chi\left(S^{1} \times{ }_{\tau} \Sigma\right)$, the Chern-Simons invariant $\operatorname{CS}(\alpha)=0$.

The $\rho$-invariant $\rho_{\mathrm{ad} \alpha_{t}}$ is also constant along paths $\alpha_{t}$ of flat connections lying in a single stratum of $\chi$, but in general it may jump as a path descends into a lower stratum - cf. [14,27]. The character variety of $S^{1} \times_{\tau} \Sigma$ is not smooth, but it turns out that the $\rho$-invariant doesn't change.

Lemma 1.9. For any $\alpha \in \chi\left(S^{1} \times_{\tau} \Sigma\right)$, the invariant $\rho_{\text {ad } \alpha}\left(S^{1} \times_{\tau} \Sigma\right)=0$.

Proof. Because the $\rho$-invariant is locally constant on strata, it suffices to check the vanishing for a single representation in each component of the three strata of $\chi\left(S^{1} \times_{\tau} \Sigma\right)$. The technique is the same in each stratum, so we just check the case when the restriction to $S^{1} \times_{\tau} \Sigma$ is irreducible. Let $\alpha$ be a representation sending $a_{1} \rightarrow \imath, a_{2} \rightarrow \jmath$, and $b_{1}, b_{2} \rightarrow 1$. There are two choices $( \pm k)$ for $\alpha(z)$; the argument works with either one. Notice that $\alpha_{\Sigma}$ extends over the obvious genus2 handlebody $C$ with boundary $\Sigma$, so that $\alpha$ extends over the (4-dimensional) mapping torus $S^{1} \times{ }_{\tau} C$.

Essentially by definition [3]

$$
\rho_{\text {ad } \alpha}\left(S^{1} \times_{\tau} \Sigma\right)=3 \operatorname{sign}\left(S^{1} \times{ }_{\tau} C\right)-\operatorname{sign}\left(S^{1} \times{ }_{\tau} C ; \operatorname{ad} \alpha\right)
$$


Since $\tau_{*}=-1$ on $H_{1}(C)$, a simple Wang sequence shows that $H_{2}\left(S^{1} \times_{\tau} C\right)=0$, so the first signature vanishes. To compute the second signature, we compare the cohomology of $S^{1} \times_{\tau} C$ with that of $S^{1} \times{ }_{\tau} \Sigma$. Both of these are computed via Wang sequences, summarized in the following diagram in which ad $\alpha$-coefficients are understood.

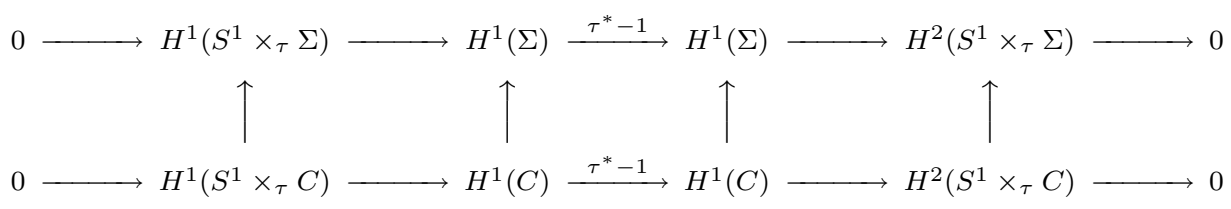

Recalling that $\alpha$ is irreducible on $\Sigma$, we have that $H^{2}(\Sigma ; \operatorname{ad} \alpha)=H^{0}(\Sigma ; \operatorname{ad} \alpha)$ vanishes. Similarly, $H^{2}(C ; \operatorname{ad} \alpha)=H^{0}(C ; \operatorname{ad} \alpha)$. One can compute that $\tau^{*}$ is the identity, using group cohomology. Alternatively, since $H^{1}(\Sigma ; \operatorname{ad} \alpha)$ is the tangent space to $\chi\left(\pi_{1}(\Sigma)\right)$, on which $\tau$ acts by the identity, $\tau^{*}=i d$. A similar remark applies to the action on $H^{1}(C$; ad $\alpha)$. Since $C$ has the homotopy type of 1-complex, $0=H_{2}(C ; \operatorname{ad} \alpha) \cong H^{1}(C, \Sigma ; \operatorname{ad} \alpha)$, and $H^{1}(C ; \operatorname{ad} \alpha) \rightarrow H^{1}(\Sigma ; \operatorname{ad} \alpha)$ is an injection. A diagram chase shows that $H^{2}\left(S^{1} \times_{\tau} C\right.$; ad $\left.\alpha\right)$ injects into $H^{2}\left(S^{1} \times_{\tau} \Sigma ;\right.$ ad $\left.\alpha\right)$, which implies that the second signature vanishes as well.

The vanishing of $\operatorname{CS}(\alpha)$ and $\rho_{\mathrm{ad} \alpha}$ could equally well have been obtained using the Seifert-fibered structure of $S^{1} \times_{\tau} \Sigma$, as in [16]. Alternatively, the jumps in the $\rho$-invariant could presumably be calculated using the techniques of $[14,27]$.

\subsection{Perturbations}

It is not necessarily the case that the flat connections on $M$ form a smooth 0dimensional variety. If they don't, then it is necessary to perturb the flatness equations, in order to define the Floer homology groups. For the purposes of this paper, it is desirable to make the perturbations in such a way that perturbed-flat connections can be cut and pasted along $\Sigma$. Now a standard method (compare $[41,18])$ for perturbing the equation $F_{A}=0$ is to replace the right side by an $s u_{2}$-valued 2-form supported in a neighborhood of a link $L \subset M$. A suitable class of 2-forms can be defined as follows: Choose first a link $L$ in $M$, and for each component $L_{i}$ a $C^{2}$ function $\bar{h}_{i}:[-2,2] \rightarrow \mathbf{R}$. The whole collection determines a function on the space of connections

$$
h(A)=\sum_{i} \int_{D^{2}} \bar{h}_{i}\left(\operatorname{tr} \operatorname{hol}_{L_{i}}(x, A)\right) \eta d x
$$

where $\eta$ is a bump function on the normal disk $D^{2}$ and $\operatorname{hol}_{L_{i}}(x, A)$ denotes the holonomy around a curve in $L_{i} \times D^{2}$. 
Any such $h$, as a function on the space of connections, has a gradient $\nabla h(A)$, which is naturally a 1 -form on $M$, supported near $L$. The solutions to the equation $F_{A}=* \nabla h(A)$ are called $h$-flat connections, and it can be shown that for a sufficiently complicated link $L$, a generic choice of $h$ will result in a smooth moduli space $\chi_{h}$ of $h$-flat connections. By construction, the $h$-flat connections are the critical points of the function $\mathrm{CS}_{h}=\mathrm{CS}-h$.

In order to cut/paste an $h$-flat connection along $\Sigma$, it is necessary that the link $L$ along which the perturbation is supported be disjoint from $\Sigma$. (In other words, if $L$ hits $\Sigma$, the $h$-flat connections on $\Sigma$ don't enjoy a symmetry property analogous to 1.1.) Recall that $M$ is divided into two pieces $A$ and $B$. If $L$ is disjoint from $\Sigma$, then we can consider separately the $h$-flat connections on $A$ and $B$. Such connections can clearly be glued up exactly when they agree on $\Sigma$. In other words, $\chi_{h}(M)$ is (essentially) the fiber product of

$$
\chi_{h}(A) \times_{\chi(\Sigma)} \chi_{h}(B)
$$

(This description must be modified, in a standard way, when connections are reducible along $\Sigma$.) In particular, the correspondence between flat connections on $M$ and $M^{\tau}$ continues to hold for $h$-flat connections which are flat along $\Sigma$.

Theorem 1.10. Let $M$ be a homology sphere, and let $\Sigma$ be an embedded genus-2 surface. Then there is a link $L$ in the complement of $\Sigma$, and a perturbation of the equation $F_{A}=0$ to an equation of the form $F_{A}=* \nabla h(A)$, where the 2-form $* \nabla h(A)$ is supported near $L$, with the following properties: For any solution $\alpha$ (i.e. $h$-flat connection), $\mathcal{H}^{*}(M ; \operatorname{ad} \alpha)=0$, and the restriction of $\alpha$ to $\Sigma$ is an irreducible flat connection.

Here $\mathcal{H}^{j}(M ; \operatorname{ad} \alpha)$ denotes the space of ad $\alpha$-valued harmonic $j$-forms on $M$.

We now turn to setting up the proof of Theorem 1.10. Let $r_{A}$ (resp. $r_{B}$ ) denote the restriction of connections from $A$ (resp. $B$ ) to $\Sigma$. Finding a perturbation $h$ for which $\chi_{h}(M)$ is smooth breaks into two steps: smoothness for the two sides, and transversality of the maps $r_{A}$ and $r_{B}$. We will treat these issues using results from the paper of C. Herald [22].

Following that paper, let $\mathcal{M}(X)$ denote the flat SU-connections on a manifold $X$, and $\mathcal{M}^{\mathbf{Z}_{2}}$ (resp. $\mathcal{M}^{\mathrm{U}(1)}, \mathcal{M}^{\mathrm{SU}(2)}$ ) denote the connections with stabilizer $\mathbf{Z}_{2}$ (resp. U(1), $\mathrm{SU}(2)$ ). If $X$ is a manifold with boundary, and $G \subset H \subset \mathrm{SU}(2)$, then $\mathcal{M}^{G, H}$ consists of connections with stabilizer (on $X$ ) equal to $G$ and stabilizer of the restriction to $\partial X$ equal to $H$. For a generic perturbation $h, \mathcal{M}_{h}(X)$ will be a stratified space, with strata indexed by the various possible pairs $(G, H)$. In the case when $X=A$ or $B$, so that $\partial X=\Sigma$ is a surface of genus 2, not all possible combinations of $(G, H)$ occur as strata of $\mathcal{M}_{h}(X)$, after the perturbation: Only the $\left(\mathbf{Z}_{2}, \mathbf{Z}_{2}\right),\left(\mathbf{Z}_{2}, U(1)\right),(U(1), U(1))$, and $(\mathrm{SU}(2), \mathrm{SU}(2))$ strata will appear.

The paper of Herald gives some additional results pertaining to the restriction map $r_{A}$ from the various strata $\mathcal{M}_{h}^{G, H}(A)$ to $\mathcal{M}_{h}^{H}(\Sigma)$. In rough terms, Herald's 
paper shows that, for generic perturbations of the equations on $A$ and $B$, the maps $r_{A}$ and $r_{B}$ are transverse. Of course, since all the spaces involved are, at best, stratified, the transversality must be taken in a suitable sense. Precise statements are given in Lemma 33 and Proposition 34 of [22]. From these results, we will prove:

Lemma 1.11. Suppose that $M$ is a homology sphere. Then for a generic perturbation $h$ of the equations, the images of the restriction maps

$$
\mathcal{M}_{h}^{G, H}(A) \stackrel{r_{A}}{\longrightarrow} \mathcal{M}^{H}(\Sigma) \stackrel{r_{B}}{\longleftarrow} \mathcal{M}_{h}^{G^{\prime}, H}(B)
$$

are empty, except if $(G, H)=\left(G^{\prime}, H\right)=\left(\mathbf{Z}_{2}, \mathbf{Z}_{2}\right)$ or $(\mathrm{SU}(2), \mathrm{SU}(2))$. In the $\left(\mathbf{Z}_{2}, \mathbf{Z}_{2}\right)$ case, the maps will be transverse, while in the $(\mathrm{SU}(2), \mathrm{SU}(2))$ case the intersection is isolated at the trivial connection.

Proof. According to [22, Theorem 15], generic perturbations of the equations on $A$ and $B$ will eliminate all but the $\left(\mathbf{Z}_{2}, \mathbf{Z}_{2}\right),\left(\mathbf{Z}_{2}, \mathrm{U}(1)\right),(\mathrm{U}(1), \mathrm{U}(1))$, and $(\mathrm{SU}(2), \mathrm{SU}(2))$ strata. Evidently, connections with the same restriction to $\Sigma$ must have the same stabilizer on $\Sigma$, so there are a limited number of cases to examine. Here is the full list of possibilities (modulo switching the letters $A$ and $B$ ), together with a description of what happens in each case:

1. $r_{A}\left(\mathcal{M}_{h}^{\mathrm{SU}(2), \mathrm{SU}(2)}(A)\right) \cap r_{B}\left(\mathcal{M}_{h}^{\mathrm{SU}(2), \mathrm{SU}(2)}(B)\right)=\{\Theta\}$ : When $h=0$, the only points in the fiber product would be the flat connections with image of the holonomy in $\mathbf{Z}_{2}$. But since $M$ is a homology sphere, the only possibility is the trivial connection, which is an isolated point in $\chi(M)$ (cf. [1]). This situation is stable under small perturbations, so remains true for the perturbed moduli space.

2. $r_{A}\left(\mathcal{M}_{h}^{\mathrm{U}(1), \mathrm{U}(1)}(A)\right) \cap r_{B}\left(\mathcal{M}_{h}^{\mathrm{U}(1), \mathrm{U}(1)}(B)\right)=\emptyset$ : This intersection is empty before the perturbation, since $M$ is a homology sphere, and so remains empty if $h$ is sufficiently small.

3. $r_{A}\left(\mathcal{M}_{h}^{\mathbf{Z}_{2}, \mathrm{U}(1)}(A)\right) \cap r_{B}\left(\mathcal{M}_{h}^{U(1), U(1)}(B)\right)=\emptyset$ : $\quad$ The image of $\mathcal{M}_{h}^{U(1), U(1)}(B)$ is a 2-dimensional submanifold of the smooth, 4-dimensional manifold $\mathcal{M}^{\mathrm{U}(1)}(\Sigma)$. Theorem 15 of Herald's paper says that if $\mathrm{h}$ is generic, then $\mathcal{M}^{\mathbf{Z}_{2}, \mathrm{U}(1)}(A)$ is 0-dimensional, so its image under $r_{A}$ is a finite set of points in $\mathcal{M}^{\mathrm{U}(1)}(\Sigma)$. Moreover, Lemma 33 of [22] says that the restriction map from an appropriate subset of $\{$ connections $\} \times\{$ perturbations $\}$ to $\mathcal{M}^{\mathrm{U}(1)}(\Sigma)$ is a submersion. This means that $r_{A}\left(\mathcal{M}_{h}^{\mathbf{Z}_{2}, \mathrm{U}(1)}(A)\right)$ can be moved arbitrarily in $\mathcal{M}^{\mathrm{U}(1)}(\Sigma)$, and so can be arranged to miss $r_{B}\left(\mathcal{M}_{h}^{\mathrm{U}(1), \mathrm{U}(1)}(B)\right)$.

4. $r_{A}\left(\mathcal{M}^{\mathbf{Z}_{2}, \mathrm{U}(1)}(A)\right) \cap r_{B}\left(\mathcal{M}^{\mathbf{Z}_{2}, U(1)}(B)\right)=\emptyset$ : This is the same argument: the 
two finite sets of points can be made disjoint by a small perturbation.

5. $r_{A}\left(\mathcal{M}_{h}^{\mathbf{Z}_{2}, \mathbf{Z}_{2}}(A)\right) \pitchfork r_{B}\left(\mathcal{M}_{h}^{\mathbf{Z}_{2}, \mathbf{Z}_{2}}(B)\right)=$ a finite set of points: As in the previous two items; the transversality follows from [22, Lemma 33].

Proof of Theorem 1.10. Choose a perturbation $h$ as described in Lemma 1.1. Then $\mathcal{M}_{h}(M)$, being the fiber product of $\mathcal{M}_{h}(A)$ and $\mathcal{M}_{h}(B)$, will consist of the (isolated) trivial connection, together with the fiber product of the $\left(\mathbf{Z}_{2}, \mathbf{Z}_{2}\right)$ strata. A final application of [22, Lemma 33] shows that the maps $r_{A}$ and $r_{B}$

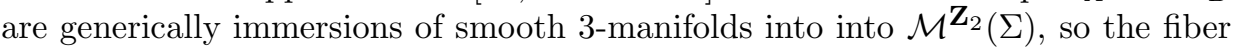
product is a finite set of points. The maps on harmonic forms induced by $r_{A}$ and $r_{B}$ are just the differentials of those maps. So the fact that the cohomology at any point in the fiber product is trivial is simply a restatement of the transversality condition.

As described in [7, §3.3], the perturbed equations on $M$ extend to perturbed anti-self-duality equations on $Y^{\tau}$. Here, the perturbation is supported on a neighborhood of $L \times \mathbf{R} \subset Y_{\infty}^{\tau}$, and is hence a 'time independent' deformation of the ASD equations, in the terminology of [7]. (The notation $Y_{\infty}^{\tau}$ indicates that halfinfinite tubes are added along the boundary components of $Y^{\tau}$.) In our situation, the solutions will be flat on the complement of the neighborhood $\nu(L \times \mathbf{R})$, and so we will continue to refer to them as $h$-flat connections.

Definition 1.12. The moduli space of $h$-ASD orbifold $\mathrm{SO}(3)$ connections on $Y_{\infty}^{\tau}$, with exponential decay to $h$-flat connections $\alpha, \beta$ on $M$ and $M^{\tau}$, respectively, will be denoted by

$$
\mathcal{M}_{h}\left(Y^{\tau} ; \alpha, \beta\right)
$$

If, as in the corollary below, the Stiefel-Whitney class $w$ or the charge $c$ is specified in advance, the notation will be expanded to $\mathcal{M}_{h, c}^{w}$.

Corollary 1.13. Let $M$ be a homology sphere containing a genus-2 surface $\Sigma$. Let $* \nabla h(A)$ be a 2-form supported along a link $L$ in the complement of $\Sigma$, having the properties described in Theorem 1.10. Then for any $h$-flat connection on $M$, there is a unique extension to an $h$-flat orbifold connection on $Y^{\tau}$. (The behavior at the singular points, and $w=w_{2}$ of the bundle are as specified in Theorem 1.6.) Restricting to the boundary gives a one-to-one correspondence

$$
\mathcal{M}_{h}(M) \leftrightarrow \mathcal{M}_{h, 0}^{w}\left(Y^{\tau}\right) \leftrightarrow \mathcal{M}_{h}\left(M^{\tau}\right)
$$

Proof of corollary. Apply the constructions of section 1.2 to the 3-manifold $N$ obtained by removing a tubular neighborhood of $L$ from $M$. (Note that the arguments in section 1.2 did not assume that $M$ was closed, and hence apply without 
change to $N$.) The result is a cobordism from $N$ to $N^{\tau}$, obtained by removing a tubular neighborhood of $L \times \mathbf{R}$ from $Y^{\tau}$. By Theorem 1.6, there are one-to-one correspondences between $\chi(N)$, the flat orbifold connections on $Y^{\tau}-\nu(L \times \mathbf{R})$, and $\chi\left(N^{\tau}\right)$. The argument that a flat connection on $M-\nu(L)$ extends uniquely to an $h$-flat connection on $M$ applies in the 4-dimensional situation, and shows that a flat connection on $Y^{\tau}-\nu(L \times \mathbf{R})$ extends uniquely to an $h$-flat connection on $Y^{\tau}$.

The $h$-flat extension defines the unique element of $\mathcal{M}_{h, 0}^{w}\left(Y^{\tau} ; \alpha, \alpha^{\tau}\right)$. We will show later that the formal dimension of this moduli space is 0 ; we need to know that the one point in the moduli space is a smooth point. The usual deformation theory says that this will follow from the following lemma.

Lemma 1.14. Let $A$ be the unique element of $\mathcal{M}_{h, 0}^{w}\left(Y^{\tau} ; \alpha, \alpha^{\tau}\right)$. Then the space of harmonic forms $\mathcal{H}^{1}\left(Y^{\tau}\right.$; ad $\left.A\right)$ vanishes.

Proof. In the unperturbed situation (i.e. if $\alpha$ were a smooth isolated flat connection), this could be readily proved by interpreting $\mathcal{H}^{1}\left(Y_{\infty}^{\tau} ;\right.$ ad $\left.A\right)$ as a twisted cohomology group, and then computing topologically, using the isomorphisms $H^{1}(M ; \operatorname{ad} \alpha) \stackrel{i^{*}}{\leftarrow} \mathcal{H}^{1}\left(Y^{\tau} ; \operatorname{ad} A\right) \stackrel{i^{*}}{\rightarrow} H^{1}\left(M^{\tau} ; \operatorname{ad} \alpha^{\tau}\right)=0$. Equivalently, one could interpret the cohomology group in terms of group cohomology, and use the presentation (2) of $\pi_{1}\left(W^{\tau}\right)$ to obtain the same isomorphism. Note that either of these arguments would apply to $Y^{\tau}-\nu(L \times \mathbf{R})$. The proof of the lemma would thus be completed if there were an appropriate Mayer-Vietoris principle for $\operatorname{ad}(A)$-valued harmonic forms on manifolds such as $Y_{\infty}^{\tau}$. Here it would have to be applied to the decomposition of $Y^{\tau}$ into $Y^{\tau}-\nu(L \times \mathbf{R}) \cup \nu(L \times \mathbf{R})$. While such an argument would undoubtedly succeed, we know of no convenient reference, and proceed somewhat differently.

It is explained carefully in Appendix A of [10] that the (exponentially decaying) harmonic forms on $Y_{\infty}^{\tau}$ can be identified with harmonic forms on $Y^{\tau}$ (regarded as having a long, but finite, collar on its boundary.) Double $Y^{\tau}$ along its boundary, to obtain an orbifold $Z$, which contains a copy of $\nu\left(L \times S^{1}\right)$. By a Mayer-Vietoris argument, found in $\left[10\right.$, Appendix B], the harmonic forms $\mathcal{H}^{1}\left(Y^{\tau} ;\right.$ ad $\left.A\right) \cong \mathcal{H}^{1}(Z ;$ ad $A)$, so it suffices to show the latter. But now we can use the Mayer-Vietoris principle for

$$
Z=\left(Z-\nu\left(L \times S^{1}\right)\right) \cup \nu\left(L \times S^{1}\right)
$$

As remarked above, the restriction $\mathcal{H}^{1}\left(Z-\nu\left(L \times S^{1}\right) ; \operatorname{ad} A\right) \rightarrow \mathcal{H}^{1}(M-\nu(L) ; \operatorname{ad} A)$ is an isomorphism, as is the restriction $\mathcal{H}^{1}\left(\nu\left(L \times S^{1}\right) ; \operatorname{ad} A\right) \rightarrow \mathcal{H}^{1}(\nu(L) ; \operatorname{ad} A)$. By a diagram chase, the vanishing of the harmonic forms for $Z$ follows from the same on $M$, plus the Mayer-Vietoris principle for forms on $M=(M-\nu(L)) \cup \nu(L)$. 


\subsection{Spectral flow}

In this section we show that corresponding $h$-flat connections in $\chi_{h}(M)$ and $\chi_{h}\left(M^{\tau}\right)$ have the same grading in Floer homology. As remarked at the end of section 1.2, for connections which are actually flat, this can be proved using the Atiyah-Patodi-Singer index theorem. (One would need, in addition to the $\rho$ and CS invariants computed there, a calculation of the twisted signature of $W^{\tau}$.) This approach is not workable for the $h$-flat connections because it would involve a direct calculation of the index of a Dirac-type operator, whose kernel and cokernel are not topological invariants. An alternative technique, which we adopt, is to use the definition of the grading in terms of spectral flow of paths of differential operators. Given the splitting of $M$ along $\Sigma$ into pieces $A$ and $B$, work of Cappell, Lee, and Miller $[9,10,11]$ (and others $[13,36]$ ) calculates the spectral flow on $M$ in terms of spectral flows of operators on $A$ and $B$, and an 'interaction term' called the Maslov index. We will show that the these terms do not change in passing from $\alpha$ to $\alpha^{\tau}$.

Suppose that $M$ has a Riemannian metric, which for future reference will be chosen to be a product $\Sigma \times[-r, r]$ near $\Sigma$. Here $r$ is chosen sufficiently large, so that Theorem $\mathrm{C}$ of [11] applies to all paths of connections under consideration. Let $\alpha$ be a connection on $M$ and choose a path $\alpha_{t}$ of connections from the trivial connection $\Theta_{M}$ to $\alpha$. For each $\alpha_{t}$ there is defined a first-order elliptic operator

$$
D_{\alpha_{t}}=\left(\begin{array}{cc}
0 & d_{\alpha_{t}}^{*} \\
d_{\alpha_{t}} & -* d_{\alpha_{t}}
\end{array}\right): \underset{\Omega^{1}\left(M ; \operatorname{ad} \alpha_{t}\right)}{\oplus} \rightarrow \begin{gathered}
\Omega^{0}\left(M ; \operatorname{ad} \alpha_{t}\right) \\
\Omega^{1}\left(M ; \operatorname{ad} \alpha_{t}\right)
\end{gathered}
$$

The Floer grading of $\alpha$ is then given by the spectral flow of the path of operators $\left\{D_{\alpha_{t}} \mid t \in[0,1]\right\}$. To be more precise, it is the $(-\epsilon, \epsilon)$-spectral flow in the terminology of [11], i.e. the intersection number of the graph of the eigenvalues of $D_{\alpha_{t}}$ in $[0,1] \times \mathbf{R}$ with the line from $(0,-\epsilon)$ to $(1, \epsilon)$. The work of Cappell-Lee-Miller actually computes the $(\epsilon, \epsilon)$-spectral flow, which we will denote by $\mathrm{SF}^{\epsilon}$. Fortunately, it is not hard to account for the difference between the two spectral flows.

Lemma 1.15. Let $\epsilon>0$, and suppose that $D_{t}$ be a path of self-adjoint operators, such that any nonzero eigenvalue $\lambda$ of $D_{0}$ or $D_{1}$ satisfies $\lambda>\epsilon$. Then the $(-\epsilon, \epsilon)$ spectral flow $\mathrm{SF}\left(D_{t}\right)$ and the $(\epsilon, \epsilon)$ spectral flow $\mathrm{SF}^{\epsilon}\left(D_{t}\right)$ are related by

$$
\mathrm{SF}\left(D_{t}\right)=\stackrel{\epsilon}{\mathrm{S}}\left(D_{t}\right)+\operatorname{dim}\left(\operatorname{ker}\left(D_{0}\right)\right)
$$

Remark 1.15. As in [11], the hypothesis of the lemma will hold if $\epsilon$ is chosen to be $1 / r^{2}$ for a sufficiently large value of $r$.

Proof. The hypothesis implies that a 0 -eigenvalue of $D_{0}$ either flows to a positive eigenvalue $>\epsilon$ of $D_{1}$, to a 0 -eigenvalue, or to a negative eigenvalue $<-\epsilon$. In 
the first case, it contributes +1 to $\mathrm{SF}^{\epsilon}$ and 0 to $\mathrm{SF}$. In the latter two cases, it contributes -1 to $\mathrm{SF}$, and 0 to $\mathrm{SF}^{\epsilon}$, and the result follows since all other paths of eigenvalues contribute the same to both counts of spectral flow. Another way to phrase the argument is that any path must have 0 intersection number of with the (oriented) triangle in the $(t, \lambda)$-plane with vertices $(0,-\epsilon),(0, \epsilon)$, and $(1, \epsilon)$. From this point of view, the lemma states the obvious fact that the intersection with the piece along the $\lambda$-axis is given by $\operatorname{dim}\left(\operatorname{ker}\left(D_{0}\right)\right)$.

The spectral flow $\operatorname{SF}_{M}\left(D_{\Theta}, D_{\alpha}\right)$ being independent of the choice of path means that we can choose a path of connections which is well-suited to cutting and pasting.

Construction 1.16. Recall from Theorem 1.10 that the $h$-flat connection $\alpha$ is flat and irreducible on $\Sigma$. Since $\chi(\Sigma)$ is connected, there is a smooth path $\alpha_{\Sigma, t}$ of flat connections on $\Sigma$, with $\alpha_{\Sigma, 0}$ the trivial connection. It is convenient to choose the path so that $\alpha_{\Sigma, t}$ is irreducible for $0<t \leq 1$. This may be done since the space of irreducible flat connections on $\Sigma$ is connected. One consequence of this choice is that the kernel of the 'tangential' operator $\hat{D}_{\alpha_{t}}$, given by $H^{0}\left(\Sigma\right.$, ad $\left.\alpha_{t}\right) \oplus$ $H^{0}\left(\Sigma\right.$, ad $\left.\alpha_{t}\right) \oplus H^{1}\left(\Sigma\right.$, ad $\left.\alpha_{t}\right)$, is constant for $t>0$. Using a partition of unity, extend $\left\{\alpha_{\Sigma, t}\right\}$ to a path of connections on $M$ with $\alpha_{0}$ the trivial connection. The connections may be assumed to be flat, and pulled back from $\Sigma$, on the tube $[-r, r] \times \Sigma$.

By Lemma 1.1, there is an element $g_{t} \in \mathrm{SU}(2)$ conjugating $\alpha_{\Sigma, t} \circ \tau_{*}$ to $\alpha_{\Sigma, t}$. Since $\alpha_{\Sigma, t}$ is irreducible, $g_{t}$ is determined up to sign, and so the induced path in $\mathrm{SO}(3)$ is smooth. By path-lifting for the double covering $\mathrm{SU}(2) \rightarrow \mathrm{SO}(3)$, the path $g_{t}$ may thus be assumed to be smooth. By choosing the path to agree with some previously specified model path near $t=0$, we can arrange that the path has a smooth extension to $t=0$.

The result of this construction is that each element in the path $\alpha_{t}$ can be cut and pasted to give a smooth path of connections $\alpha_{t}^{\tau}$ on $M^{\tau}$, giving rise to paths of operators $D_{\alpha_{t}}$ and $D_{\alpha_{t}^{\tau}}$ on $M$ and $M^{\tau}$. The splitting technique for computing the spectral flow of $D_{\alpha_{t}}$ and $D_{\alpha_{t}^{\tau}}$ involves the restriction of $s u_{2}$-valued forms (and operators on these spaces) on $M$ to those on $A$ and $B$ and subsequently to $\Sigma$. We make the following convention:

Convention 1.17. Forms on $A, B$ and $\Sigma$, all viewed as submanifolds of $M^{\tau}$, are identified with the same forms when $A, B$ and $\Sigma$ are viewed as submanifolds of $M$.

The difference between gluing $A$ to $B$ via $\tau$ and via the identity is encoded in the restriction maps from forms on $A$ or $B$ to those on $\Sigma$. By construction, $A$ as a submanifold of $M^{\tau}$ is identified with $A$ as a submanifold of $M$, and under this 
identification, $\alpha_{A}^{\tau}$ is identified with $\alpha_{A}$. In this way, the restriction map

$$
\Omega^{*}\left(A ; \operatorname{ad} \alpha_{t}^{\tau}\right) \rightarrow \Omega^{*}\left(\Sigma ; \operatorname{ad} \alpha_{t}^{\tau}\right)
$$

is the same as the restriction map

$$
\Omega^{*}\left(A ; \operatorname{ad} \alpha_{t}\right) \rightarrow \Omega^{*}\left(\Sigma ; \operatorname{ad} \alpha_{t}\right) .
$$

In contrast, the restriction map from forms on $B$ (viewed as a subset of $M^{\tau}$ ) to forms on $\Sigma$ is given by the composition

$$
\Omega^{*}\left(B ; \operatorname{ad} \alpha_{t}\right) \stackrel{r_{B}^{*}}{\rightarrow} \Omega^{*}\left(\Sigma ; \operatorname{ad} \alpha_{t}\right) \stackrel{\tau^{*}}{\rightarrow} \Omega^{*}\left(\Sigma ; \operatorname{ad} \alpha_{t}\right)
$$

where $r_{B}^{*}$ is the restriction map from forms on $B \subset M$ to forms on $\Sigma$ and $\hat{\tau}^{*}$ is induced by $\tau$ as described in equation (1) at the beginning of section 1. (Note that $\hat{\tau}^{*}$ actually depends on $t$, but this dependence will be suppressed in the notation.)

The operators $D_{\alpha_{t}}(A)$ and $D_{\alpha_{t}^{\tau}}(A)$, obtained by restricting from $M$ and $M^{\tau}$ to $A$ are identical, as are the the restrictions $D_{\alpha_{t}}(B)$ and $D_{\alpha_{t}^{\tau}}(B)$. It is important to remark, however, that because of the action of $\hat{\tau}^{*}$ on $\Omega^{*}(\Sigma$; ad $\alpha)$, the boundaryvalue problems on $B$ associated to the two operators are not a priori the same.

We now summarize the splitting results from $[10,11]$ which will be used to compare the spectral flow of the path $\alpha_{t}$ with that of its mutated cousin. As described in the paragraphs before Theorem $\mathrm{C}$ in [11], divide the interval $[0,1]$ into sub-intervals $0=a_{0}<a_{1} \ldots a_{n}=1$ with the property that for $t \in\left[a_{i}, a_{i+1}\right]$ there are no eigenvalues of $\hat{D}_{\alpha_{t}}$ in the intervals $\left(K_{i}, K_{i}+\delta\right)$ and $\left(-K_{i}-\delta,-K_{i}\right)$, for some positive $K_{i}$ and $\delta$. The spectral flow $\mathrm{SF}^{\epsilon}$ is then the sum of the spectral flows on the subintervals, so it suffices to compare on the intervals $\left[a_{i}, a_{i+1}\right]$.

For $t$ in such an interval, there are smoothly varying Atiyah-Patodi-Singer type boundary conditions for the $D_{\alpha_{t}}(A)$ and $D_{\alpha_{t}}(B)$ (i.e. the restriction of $D_{\alpha_{t}}$ to $A$ (resp. $B)$ ), described as follows. The finite dimensional space $\mathcal{H}\left(t, K_{i}\right) \subset$ $\Omega^{0}\left(\Sigma ;\right.$ ad $\left.\alpha_{t}\right) \oplus \Omega^{1}\left(\Sigma ;\right.$ ad $\left.\alpha_{t}\right)$ is defined to be the span of the eigenfunctions of $\hat{D}_{\alpha_{t}}$ with eigenvalue less than $K_{i}$ in absolute value. Note that the images of $\operatorname{ker}\left(D_{\alpha_{t}}(A)\right)\left(\right.$ resp. $\left.\operatorname{ker}\left(D_{\alpha_{t}}(B)\right)\right)$ under restriction to $\Sigma$ give Lagrangian subspaces $L_{t}(A)\left(\right.$ resp. $\left.L_{t}(B)\right)$ in $\operatorname{ker}\left(\hat{D}_{\alpha_{t}}\right)$. These are extended to Lagrangian subspaces of $\mathcal{H}\left(t, K_{i}\right)$ by defining

$$
\begin{aligned}
& \mathcal{L}_{t}(A)=L_{t}(A) \oplus\left[P_{+}(t) \cap \mathcal{H}\left(t, K_{i}\right)\right] \\
& \mathcal{L}_{t}(B)=L_{t}(B) \oplus\left[P_{-}(t) \cap \mathcal{H}\left(t, K_{i}\right)\right]
\end{aligned}
$$

where $P_{ \pm}(t)$ are the sums of the positive/negative eigenspaces of $\hat{D}_{t}$. The resulting path (for $t \in\left[a_{i}, a_{i+1}\right]$ ) of pairs of Lagrangian subspaces defines (cf. [9]) a Maslov index

$$
\operatorname{Mas}\left(\mathcal{L}_{t}(A), \mathcal{L}_{t}(B)\right) \in \mathbf{Z}
$$


The operator $D_{\alpha_{t}}(A)$ (for $t \in\left[a_{i}, a_{i+1}\right]$ ) is Fredholm on the domain consisting of forms in $L_{1}^{2}\left(\Omega^{0}\left(A ; \operatorname{ad} \alpha_{t}\right) \oplus \Omega^{1}\left(A ; \operatorname{ad} \alpha_{t}\right)\right)$ whose restriction to $\Sigma$ lie in

$$
\mathcal{L}_{t}(A) \oplus P_{+}\left(t, K_{i}\right) .
$$

Here $P_{+}\left(t, K_{i}\right)$ denotes the span of the eigenfunctions of $\hat{D}_{\alpha_{t}}$ with eigenvalue greater than $K_{i}$. Similarly, $D_{\alpha_{t}}(B)$ is Fredholm, where now its domain is specified by the requirement that the forms, upon restriction to $\Sigma$, lie in $\mathcal{L}_{t}(B) \oplus P_{-}\left(t, K_{i}\right)$. It follows that the spectral flows $\operatorname{SF}^{\epsilon}\left(D_{\alpha_{t}}(A)\right)$ and $\operatorname{SF}^{\epsilon}\left(D_{\alpha_{t}}(B)\right)$ are defined for $t \in\left[a_{i}, a_{i+1}\right]$.

Theorem 1.18. For any irreducible $h$-flat connection $\alpha, \mathrm{SF}_{M}\left(D_{\Theta}, D_{\alpha}\right)=$ $\mathrm{SF}_{M^{\tau}}\left(D_{\Theta}, D_{\alpha^{\tau}}\right)$. Equivalently, the Floer grading of $\alpha^{\tau}$ is equal to the Floer grading of $\alpha$.

Proof. Because $M$ and $M^{\tau}$ are both homology spheres, and $\alpha_{0}$ and $\alpha_{0}^{\tau}$ are trivial connections, Lemma 1.15 implies that it suffices to show that $\mathrm{SF}_{M}^{\epsilon}\left(D_{\Theta}, D_{\alpha}\right)=$ $\mathrm{SF}_{M^{\tau}}^{\epsilon}\left(D_{\Theta}, D_{\alpha^{\tau}}\right)$. Moreover, the discussion in the preceding paragraphs implies that it suffices to prove this equality when $t$ ranges over the interval $\left[a_{i}, a_{i+1}\right]$.

Theorem C of [11] states that for sufficiently small $\epsilon$,

$$
\begin{aligned}
\mathrm{SF}_{M}^{\epsilon}\left(D_{\alpha_{t}}\right)= & \mathrm{SF}^{\epsilon}\left(D_{\alpha_{t}}(A)\right)+\mathrm{SF}^{\epsilon}\left(D_{\alpha_{t}}(B)\right) \\
& +\operatorname{Mas}\left(\mathcal{L}_{t}(A), \mathcal{L}_{t}(B)\right)+\frac{1}{2}\left[\operatorname{dim} \operatorname{ker} \hat{D}\left(a_{i+1}\right)-\operatorname{dim} \operatorname{ker} \hat{D}\left(a_{i}\right)\right]
\end{aligned}
$$

where all of the terms are calculated on the interval $\left[a_{i}, a_{i+1}\right]$. Hence it suffices to show that the terms on the right hand side in the analogous formula $\left(*^{\tau}\right)$ for $\mathrm{SF}_{M^{\tau}}^{\epsilon}\left(D_{\alpha_{t}^{\tau}}\right)$ are the same as those above.

Using the convention 1.17, the operator $\hat{D}_{\alpha^{\tau}}$, when $\Sigma$ is viewed as a submanifold of $M^{\tau}$ is identified with $\hat{D}_{\alpha}$ (for $\Sigma$ viewed as a submanifold of $M$ ). Hence the kernel of $\hat{D}_{\alpha}$ is unchanged when $\alpha$ is replaced by $\alpha^{\tau}$, so the last terms in equations $(*)$ and $\left(*^{\tau}\right)$ are the same. Similarly, the Lagrangian subspace $\mathcal{L}_{t}(A)$ does not change, whether $A$ is viewed as a submanifold of $M$ or of $M^{\tau}$. It follows that $\operatorname{SF}^{\epsilon}\left(D_{\alpha_{t}}(A)\right)=\operatorname{SF}^{\epsilon}\left(D_{\alpha_{t}^{\tau}}(A)\right)$, because the two refer to spectral flow of operators which are viewed as identical.

To show that $\mathrm{SF}^{\epsilon}\left(D_{\alpha_{t}}(B)\right)$ and the Maslov index term do not change under the mutation, it suffices to show that $\hat{\tau}^{*}$ takes $\mathcal{L}_{t}(B)$ to itself. Now $\hat{\tau}^{*}$, being an automorphism of $\alpha_{t}$, commutes with $\hat{D}_{\alpha_{t}}$, and hence preserves the eigenspaces of that operator. In particular, the summand $\left[P_{-}(t) \cap \mathcal{H}\left(t, K_{i}\right)\right]$ of $\mathcal{L}_{t}(B)$ is preserved by $\hat{\tau}^{*}$, so we need to know the effect of $\hat{\tau}^{*}$ on the Lagrangian subspace $L_{t}(B) \subset$ $\operatorname{ker}\left(\hat{D}_{\alpha_{t}}\right)$.

Claim. Let $\alpha$ be a flat connection on $\Sigma$. If $\alpha$ is irreducible, so that $\operatorname{ker}\left(\hat{D}_{\alpha}\right) \cong$ $H^{1}(\Sigma ; \operatorname{ad} \alpha)$, then $\tau^{*}$ acts as the identity on $\operatorname{ker}\left(\hat{D}_{\alpha}\right)$. If $\alpha$ is $\mathrm{SU}(2)$-reducible, then

$$
\operatorname{ker}\left(\hat{D}_{\alpha}\right) \cong\left(H^{0}(\Sigma) \otimes s u_{2}\right) \oplus\left(H^{0}(\Sigma) \otimes s u_{2}\right) \oplus\left(H^{1}(\Sigma) \otimes s u_{2}\right)
$$


and $\tau^{*}$ is the identity on the first two summands and -1 on the third.

Proof of Claim. In the case that $\alpha_{\Sigma}$ is irreducible, one can show that that $\hat{\tau}^{*}$ is the identity, using group cohomology. Alternatively, since $H^{1}(\Sigma$; ad $\alpha)$ is the tangent space to the (irreducible part of) $\chi\left(\pi_{1}(\Sigma)\right)$, on which $\hat{\tau}$ acts by the identity, $\hat{\tau}^{*}=i d$. When $\alpha_{\Sigma}$ is $\mathrm{SU}(2)$-reducible, then the cohomology groups are just the ordinary de Rham cohomology groups, tensored with $s u_{2}$, and the result is trivial.

So in the case that $\alpha_{\Sigma}$ is irreducible, the invariance of $L_{t}(B)$ under $\hat{\tau}^{*}$ follows directly from the claim. In the case that $\alpha_{\Sigma}$ is $\mathrm{SU}(2)$-reducible, i.e. when $t=0$, the Lagrangian subspace $L_{0}(B)$ splits as the sum of $r_{B}^{*}\left(H^{1}(B ; \operatorname{ad} \alpha)\right)$ and the antidiagonal in $H^{0}(\Sigma$; ad $\alpha) \oplus H^{0}(\Sigma$; ad $\alpha)$. According to the claim, it is again the case that $\hat{\tau}^{*}\left(L_{0}(B)\right)=L_{0}(B)$, and the theorem follows.

The preceding argument contains most of the ingredients for comparing the Z-grading, as defined in [17], for the groups $\mathrm{HF}^{\mu}(M)$ and $\mathrm{HF}^{\mu}\left(M^{\tau}\right)$. The spectral flow defined above is defined for paths of actual connections, rather than gaugeequivalence classes of connections. An fundamental observation is that it descends to a function on $\mathcal{A} / \mathcal{G}_{0}$, where $\mathcal{G}_{0}$ is the degree-0 gauge group. Likewise, the (perturbed) Chern-Simons function on connections descends to a function $\widetilde{\mathrm{CS}}_{h}$ : $\mathcal{A} / \mathcal{G}_{0} \rightarrow \mathbf{R}$. (Given a choice of trivial connection $\Theta_{M}$, a lifting $\tilde{\alpha} \in \mathcal{A} / \mathcal{G}_{0}$ of an $h$-flat connection, and a path of connections from $\Theta_{M}$ to $\tilde{\alpha}$, the usual Chern-Weil integral over $M \times \mathbf{I}$ defines $\widetilde{\mathrm{CS}}_{h}(\tilde{\alpha})$.) Both $\mathrm{SF}$ and $\widetilde{\mathrm{CS}}_{h}$ depend on this choice of trivial connection.

Fix any choice $\Theta_{M}$, which will be used to pick out connections on all the other manifolds involved in the argument; for starters the trivial connection on $\Sigma$ will simply be the restriction of $\Theta_{M}$. Recall construction 1.16 from the discussion leading up to the proof of Theorem 1.18, in which we chose a specific path of connections $\alpha_{t}$ on $M$, which were flat and irreducible on $\Sigma$, with endpoint the trivial connection $\Theta_{M}$. Simultaneously, we chose a path of gauge transformations $g_{t}$ on $\Sigma$ with $g_{t}^{*}\left(\alpha_{t}\right)=\tau^{*}\left(\alpha_{t}\right)$. We make the convention in the subsequent discussion that any path of connections on $M$ with endpoint $\Theta_{M}$ should agree with this fixed model path near its endpoint. Note that $g_{0}$ will not be the identity gauge transformation, because $g_{t}$ is of order 4 for $t>0$.

Choose a real number $\mu$ which is not in the discrete set $\left\{\widetilde{\operatorname{CS}}_{h}(\alpha) \mid \alpha \in \chi_{h}(M)\right\}$, and for each $h$-flat connection $\alpha \in \chi_{h}(M)$, pick a representative $\tilde{\alpha}$ of its $\mathcal{G}_{0}$ orbit with $\widetilde{\mathrm{CS}}_{h}(\tilde{\alpha}) \in(\mu, \mu+1)$. The connections $\tilde{\alpha}$ form the basis of $\mathrm{CF}_{*}^{\mu}$, and the grading is defined in terms of spectral flow, where one uses the same trivial connection, and path, as were used to define the Chern-Simons invariant. (We remind the reader that the spectral flow $\operatorname{SF}\left(D_{\alpha}, D_{\beta}\right)$ changes by $8 \operatorname{deg}(g)$ when one replaces $\alpha$ by $g^{*}(\alpha)$. Hence, if we consider specific $\mathcal{G}_{0}$-representatives, the grading is actually $\mathbf{Z}$-valued, and not just $\mathbf{Z} / 8$-valued.) 
Theorem 1.19. For any $h$-flat connection $\alpha$, the $\mathbf{Z}$-grading of $\alpha^{\tau}$ is equal to the Z-grading of $\alpha$. Specifically, this means:

1. The h-flat connection $\alpha^{\tau}$ constructed by cutting and pasting satisfies $\widetilde{\mathrm{CS}}_{h}(\alpha)=$ $\widetilde{\mathrm{CS}}_{h}\left(\alpha^{\tau}\right)$.

2. If $\mu \notin\left\{\widetilde{\mathrm{CS}}_{h}(\alpha) \mid \alpha \in \chi_{h}(M)\right\}$, then $\mu \notin\left\{\widetilde{\mathrm{CS}}_{h}(\beta) \mid \beta \in \chi_{h}\left(M^{\tau}\right)\right\}$.

3. $\mathrm{SF}_{M}\left(D_{\Theta}, D_{\alpha}\right)=\mathrm{SF}_{M^{\tau}}\left(D_{\Theta}, D_{\alpha^{\tau}}\right)$

Proof. Choose a path $\alpha_{t}$ of connections on $M$ from $\Theta_{M}$ to $\alpha$, agreeing with the one from construction 1.16 near $t=0$. As was remarked earlier, the whole path can be extended to a path of connections $A_{t}$ on the basic cobordism $W^{\tau}$. The restriction of $A_{t}$ to the boundary component $M^{\tau}$ starts at a trivial connection $\Theta_{M^{\tau}}$ and ends at $\alpha^{\tau}$. Because $\alpha_{\Sigma, t}$ is flat, the restriction (say, $\alpha_{S, t}$ of $A_{t}$ to the other boundary component $S^{1} \times_{\tau} \Sigma$ is flat. However, $\alpha_{S, 0}$ is not the trivial connection, because the holonomy around $z$ is non-trivial.

In a standard way, the family of connections $A_{t}$ fits together to give a connection on the 5-manifold $W^{\tau} \times \mathbf{I}$; denote the restriction of this connection to $S^{1} \times{ }_{\tau} \Sigma \times \mathbf{I}$ by $A_{S}$. Applying Stokes' theorem to the perturbed Chern-Weil integrand

$$
\frac{1}{8 \pi^{2}} \operatorname{Tr}\left(\left(F_{A}+x\right) \wedge\left(F_{A}+x\right)\right)
$$

gives

$$
\begin{aligned}
& \widetilde{\mathrm{CS}}_{M}(\alpha)-\widetilde{\mathrm{CS}}_{M^{\tau}}\left(\alpha^{\tau}\right)=\frac{1}{8 \pi^{2}} \int_{S^{1} \times_{\tau} \Sigma \times \mathbf{I}} \operatorname{Tr}\left(F_{A_{S}} \wedge F_{A_{S}}\right) \\
& +\frac{1}{8 \pi^{2}} \int_{W^{\tau}} \operatorname{Tr}\left(\left(F_{A_{1}}+x\right) \wedge\left(F_{A_{1}}+x\right)\right)-\frac{1}{8 \pi^{2}} \int_{W^{\tau}} \operatorname{Tr}\left(\left(F_{A_{0}}+x\right) \wedge\left(F_{A_{0}}+x\right)\right)
\end{aligned}
$$

Because each connection $\alpha_{S, t}$ is flat, the first integral on the right side of this equation vanishes. Similarly, since $A_{0}$ and $A_{1}$ are $h$-flat connections, the other two integrals vanish, and the first item is proved. The second one follows immediately, and the third one is the content of Theorem 1.18.

\subsection{Invariance of $H F_{*}$ under mutation}

We can now state and prove a more precise version of Theorem 1.

Theorem 1.20. Let $M$ be an oriented homology 3-sphere, with (instanton) Floer homology $H_{*}(M)$, which contains a genus-2 surface, and let $M^{\tau}$ be the result of mutation along $\Sigma$. Let $Y^{\tau}$ be the orbifold constructed in section 1.2. Then

$$
Y_{*}^{\tau}: H F_{i}(M) \stackrel{\cong}{\rightrightarrows} H F_{i}\left(M^{\tau}\right)
$$

Similarly, if $\mu$ is a real number which is not the Chern-Simons invariant of any flat connection on $M$, then $Y_{*}^{\tau}$ induces an isomorphism on the $\mathbf{Z}$-graded instanton 
homology of Fintushel-Stern:

$$
Y_{*}^{\tau}: H F_{*}^{\mu}(M) \stackrel{\cong}{\rightrightarrows} H F_{*}^{\mu}\left(M^{\tau}\right) .
$$

Proof. Choose a perturbation, as in Theorem 1.10, so that the $h$-flat connections on $M$ and $M^{\tau}$ are all isolated smooth points. This perturbation extends to a 'time-independent' perturbation of the ASD equations on $Y^{\tau}$, and so induces a map on the $\mathbf{Z}$-graded theory as well as the usual Floer theory. Choose a metric on $Y^{\tau}$ which is generic, so that all moduli spaces on $Y^{\tau}$ are smooth of the correct dimension. Let $\alpha$ and $\beta$ be $h$-flat connections on $M$ and $M^{\tau}$, respectively. As usual in Floer theory, the map induced by $Y^{\tau}$ is given on the chain level by a matrix, whose $(\alpha, \beta)$ entry counts (with signs) the number of points in the 0 -dimensional moduli space

$$
\mathcal{M}_{h}^{w}\left(Y^{\tau} ; \alpha, \beta\right)
$$

Since $Y^{\tau}$ is an orbifold, one needs to check that $Y_{*}^{\tau}$, as defined this way, is in fact a chain map.

As usual in Floer theory, this amounts to a constraint on the possible noncompactness of 1-dimensional moduli spaces $\mathcal{M}_{h}^{w}\left(Y^{\tau} ; \alpha, \beta\right)$. One needs to see that there cannot be a 1-dimensional moduli space bubbling off at the singular points of the orbifold. Because $w_{2}$ is preserved in Uhlenbeck limits, the background connection in such a circumstance would necessarily have non-trivial holonomy at each cone point. But (cf. $[4,19,20])$ the minimum dimension for a moduli space of ASD connections on $\Sigma\left(\mathbf{R P}^{3}\right)$ is 2. From the description of the cohomology of $W^{\tau}$ in Lemma 1.3, the rational cohomology of $Y^{\tau}$ is readily obtained. Regarding $Y^{\tau}$ as a rational homology manifold, we have

$$
b_{2}^{+}\left(Y^{\tau}\right)=2, \chi\left(Y^{\tau}\right)=4, \text { and } \sigma\left(Y^{\tau}\right)=0
$$

Since $b_{2}^{+}\left(Y^{\tau}\right)>1$, reducibles may be avoided, and so $Y_{*}^{\tau}$ yields a well-defined chain map. It remains to compute the degree of $Y_{*}^{\tau}$, and to show that it induces an isomorphism on $\mathrm{HF}_{*}$ and $\mathrm{HF}_{*}^{\mu}$.

The degree is computed (compare [15]) by the formula:

$$
\operatorname{deg}\left(Y_{*}^{\tau}\right)=3\left(b_{1}\left(Y^{\tau}\right)-b_{2}^{+}\left(Y^{\tau}\right)\right)+\sum_{\text {cone points }}\left(d_{\sigma}+d_{\chi}\right)
$$

where the $d_{\sigma}$ and $d_{\chi}$ in the last sum refer to the signature and Euler characteristic defects associated to each cone point. Since the holonomy of the bundle is nontrivial at each cone point, $d_{\chi}=1$ and $d_{\sigma}=0$. From the cohomology calculations, it follows that $\operatorname{deg}\left(Y_{*}^{\tau}\right)=0$.

The $h$-flat connections yield bases for the Floer chains on $M$ and $M^{\tau}$, and $Y_{*}^{\tau}$ is described as a matrix with respect to those bases. We will show that $Y_{*}^{\tau}$, 
as a map on $\mathrm{CF}_{*}$, is represented by an upper triangular matrix, with \pm 1 along the diagonal. (The signs are presumably all 1's, but we have not checked them.) As explained in [17] (with an obvious adaptation to the orbifold setting), the $\mathbf{Z}$ grading induces an increasing filtration on $\mathrm{CF}_{*}$, and the chain map $Y_{*}^{\tau}$ respects the filtration; in matrix terms this means that $Y_{*}^{\tau}$ is upper triangular. Moreover, the proof of this statement (in Theorem 5.2 of [17]) shows that if $\alpha \in \chi_{h}(M)$ and $\beta \in \chi_{h}\left(M^{\tau}\right)$ have the same grading, then any $A$ in a 0 -dimensional moduli space $\mathcal{M}_{h}^{w}\left(Y^{\tau}\right)$ must in fact be $h$-flat. (A similar argument, in terms of an ordering on the $h$-flat connections, is presented in $[7, \S 3.1]$.) In particular, the $\left(\alpha, \alpha^{\tau}\right)$ entry is the number of $h$-flat extensions of $\alpha$ over $Y^{\tau}$, which is one. Lemma 1.14 says that this one point in $\mathcal{M}_{h}^{w}\left(Y^{\tau} ; \alpha, \alpha^{\tau}\right)$ is a smooth point, so it counts for \pm 1 . Thus $Y_{*}^{\tau}$, which is a chain map, is an isomorphism, and therefore induces an isomorphism on $\mathrm{HF}_{*}^{\mu}$ and $\mathrm{HF}_{*}$.

\section{Mutation of 4-manifolds}

The fact that the involution $\tau$ is in the center of the mapping class group of the genus-2 surface leads to two types of cutting/pasting operations along certain 3manifolds embedded in a 4-dimensional manifold $X$. Under the hypothesis that the character varieties of the 3 -manifold in question is smooth, we will show that the operation preserves the SU(2)-Donaldson invariant of $X$. Both constructions have the additional feature that it appears that gauge-theoretic invariants associated to $\mathrm{SU}(3)$-bundles should change. There is no known construction at this time for invariants associated to higher-rank bundles, unfortunately.

\subsection{Mutation along 3-manifolds of Heegaard genus-2}

The following simple lemma is well-known: see [29] and the discussion of problem 3.15 in [26].

Lemma 2.1. Let $M^{3}$ be a closed 3-manifold which admits a Heegaard splitting of genus 2 . Then there is an involution $T: M \rightarrow M$ which preserves the handlebodies and restricts to $\tau$ on the Heegaard surface $\Sigma$.

The statement of the lemma contains the proof-since $\tau$ commutes with the attaching map, its extension over the genus-2 handlebodies fits together to define an involution on $M$.

Definition 2.2. Suppose that $X$ is a 4-manifold, containing a genus-two 3-manifold $M$. Then $X^{\tau}$, the mutation of $X$ along $M$ is defined to be the result of cutting and pasting $X$ along $M$, using the involution $T$ described in the previous lemma.

The free group on two letters has the same symmetry property (Lemma 1.1) 
with regard to $\mathrm{SU}(2)$ (or $\mathrm{SO}(3)$ ) representations as does the fundamental group of a genus- 2 surface. This may be proven by computing characters, as in [40], or by the following direct argument. Write $A \in \mathrm{SU}(2)$ as $\exp (a)$, for $a \in s u_{2}$. Now any element $c \in a^{\perp} \subset s u_{2}$ of length $1 / \pi$ will have the property that

$$
\exp (c)^{-1} A \exp (c)=A^{-1}=T_{*}(A) .
$$

If $B$ is another element of $\mathrm{SU}(2)$, written as $\exp (b)$, then there is at least one element $c$ in $a^{\perp} \cap b^{\perp}$ of length $1 / \pi$. Conjugating by $\exp (c)$ will take $A \rightarrow T_{*}(A)$ and $B \rightarrow T_{*}(B)$. The same proof works for $\mathrm{SO}(3)$ representations.

Theorem 2.3. Suppose that $M$ is a genus-2 homology sphere, for which the $\mathrm{SU}(2)$ character variety $\chi(M)$ is smooth. Then for any Donaldson invariant $D_{X}$ which is defined on $X$, we have

$$
D_{X}=D_{X^{\tau}}
$$

Remark. As usual in gauge theory, the hypothesis of smoothness means that $\chi(M)$ is a smooth manifold, whose tangent space at $\alpha$ is given by $H^{1}(M ; \operatorname{ad} \alpha)$.

Proof. Under the smoothness hypothesis, it is known (compare [21, 33]) that one can express the Donaldson invariant in terms of ASD connections on the components of $X-M$, exponentially decaying to flat connections on $M$. But the symmetry property described above shows that $T_{*}$ acts trivially on the character variety $\chi(M)$, and hence does not change the gluing picture, so the Donaldson invariant does not change.

It seems reasonable that the theorem should continue to hold in the general case, when $\chi(M)$ is not discrete. One would need to first find a $T$-equivariant perturbation $h$ of the Chern-Simons invariant, and then to show that the induced action of $T$ on the $h$-flat connections is the identity. It is not hard to find an equivariant perturbation, by arranging that the link $L$ along which the perturbation is supported to be $T$-invariant. Unfortunately, we have been unable to prove that this means that the action of $T^{*}$ on $\chi_{h}(M)$ is the identity.

If there were a Donaldson-type theory for the group $\mathrm{SU}(3)$, then mutant 4manifolds would not likely have the same invariants. Here is an example, based on calculations of Hans Boden, which is waiting for an appropriate theory.

Example. Suppose that $M=\Sigma\left(a_{1}, a_{2}, a_{3}\right)$ is a Seifert-fibered space with 3 exceptional fibers. Then $M$ has a genus-2 Heegaard splitting. View $M$ as $S^{1} \times$ $\left(S^{2}-3 \operatorname{int} D^{2}\right) \cup_{3} S^{1} \times D^{2}$, where the gluing maps give rise to the multiplicity of the fibers. One of the genus-2 handlebodies is given by the union of two of the $S^{1} \times D^{2}$, together with a thickened arc joining them. (That the complement is also a handlebody is left to the reader to verify!). In $[6, \S 5.1]$, Boden shows 
that for $\Sigma(2,3,7)$ there are 4 irreducible $\mathrm{SU}(3)$ representations, two of which are the complexification of an $\mathrm{SO}(3)$ representation, and which are therefore invariant under $T_{*}$. The other two, denoted $\rho_{3}$ and $\rho_{4}$, are interchanged by $T_{*}$. One would expect that $T_{*}$ would thus act non-trivially on an SU(3) Floer-type theory. A similar phenomenon occurs for $\Sigma(2,3,6 k \pm 1)$ and presumably most Brieskorn spheres.

Remark. If the Brieskorn sphere $\Sigma$ is viewed as the link of a surface singularity (defined over the reals), then $T$ may be identified with the involution coming from complex conjugation. In particular, $T$ extends over such 4-manifolds as the Milnor fiber and canonical resolution of the singularity. So, for example, mutation of a $K 3$ surface along $\Sigma(2,3,7)$ does not produce any new 4-manifolds. As pointed out by Tom Mrowka, $T_{*}$ does not act trivially on the Floer-theory associated to the Seiberg-Witten equations. For it may be seen from the description of $T$ as complex conjugation and the computations in [34] that $T_{*}$ in fact acts as the well-known involution in the Seiberg-Witten theory.

\subsection{Mutation along genus- 2 mapping tori}

Suppose that $\varphi$ is a diffeomorphism of $\Sigma$, and form the mapping torus $S^{1} \times_{\varphi} \Sigma$. The fact that $\tau$ is in the center of the genus-2 mapping class group implies that there is an involution $T: S^{1} \times_{\varphi} \Sigma \rightarrow S^{1} \times_{\varphi} \Sigma$ whose restriction to a fiber is $\tau$. If $S^{1} \times_{\varphi} \Sigma$ is embedded in a 4-manifold $X$, then as above, we define the mutation of $X$ along $S^{1} \times{ }_{\varphi} \Sigma$ by cutting and pasting via $T$. In order to carry out the argument described above, it is important to avoid the reducible flat connections on $S^{1} \times{ }_{\varphi} \Sigma$. One way to do this is to assume that the Donaldson invariant being computed is associated to an $\mathrm{SO}(3)$ bundle with $\left\langle w_{2}, \Sigma\right\rangle \neq 0$. We need the analogue of Lemma 1.1:

Lemma 2.4. Let $T$ be the involution on $S^{1} \times_{\varphi} \Sigma$ induced by $\tau$, and let $\rho$ be an $\mathrm{SO}(3)$ representation of $\pi_{1}\left(S^{1} \times_{\varphi} \Sigma\right)$ such that $\left\langle w_{2}(\rho), \Sigma\right\rangle \neq 0$. Then $\rho \circ T_{*}$ is conjugate to $\rho$.

Proof. The fundamental group of $S^{1} \times_{\varphi} \Sigma$ has a standard presentation as

$$
\left\langle t, \pi_{1}(\Sigma) \mid t^{-1} g t=\varphi_{*}(g) \forall g \in \pi_{1}(\Sigma)\right\rangle
$$

The main point to notice is that if $\rho$ is any representation $(\mathrm{SU}(2)$ or $\mathrm{SO}(3))$, then $\rho(t)$ is determined by $\rho_{\Sigma}$, up to elements in the centralizer of $\rho\left(\pi_{1}(\Sigma)\right)$. Let $\gamma$ be any element with $\gamma^{-1} \rho_{\Sigma} \gamma=\rho_{\Sigma}$. Then it is easy to check that $\gamma^{-1} \rho(t) \gamma=h \rho(t)$ for some $h$ in the centralizer of $\rho\left(\pi_{1}(\Sigma)\right)$. In order to prove the lemma we must show that in fact $h$ is trivial. Under the hypothesis on $w_{2}$, there are only two possibilities: either the centralizer is trivial (in which case the lemma follows directly), or $\rho\left(\pi_{1}(\Sigma)\right)$ is a $\mathbf{Z}_{2} \oplus \mathbf{Z}_{2}$ subgroup, which is its own centralizer. But since every element of $\mathbf{Z}_{2} \oplus \mathbf{Z}_{2}$ has order 2, one can take $\gamma$ to be trivial, in which case it certainly conjugates $\rho(t)$ to itself. 
With this lemma in hand, the proof of Theorem 2.3 yields an analogous theorem about mutation along mapping tori.

Theorem 2.5. Suppose the mapping torus $S^{1} \times_{\varphi} \Sigma$ is embedded in $X$, and that $D^{w}$ is a Donaldson invariant associated to a bundle with $\left\langle w_{2}, \Sigma\right\rangle \neq 0$. Then $D^{w}(X)=D^{w}\left(X^{T}\right)$.

This result could apparently be deduced using techniques of V. Muñoz [35].

\section{Acknowledgement}

I would like to thank Tom Mrowka for several helpful conversations, and Hans Boden for pointing out the example discussed after theorem 2.3.

\section{References}

[1] S. Akbulut and J. McCarthy, Casson's invariant for oriented homology 3-spheres - an exposition, vol. 36 of Mathematical Notes, Princeton University Press, Princeton 1990.

[2] M. Atiyah, V. Patodi, and I. Singer, Spectral asymmetry and Riemannian geometry: I, Math. Proc. Camb. Phil. Soc. 77 (1975), 43-69.

[3] M. Atiyah, V. Patodi, and I. Singer, Spectral asymmetry and Riemannian geometry: II, Math. Proc. Camb. Phil. Soc. 78 (1975), 405-432.

[4] D. M. Austin, $\mathrm{SO}(3)$-instantons on $L(p, q) \times \mathbf{R}$, J. Diff. Geom. 32 (1990), 383-413.

[5] D. M. Austin and P. J. Braam, Equivariant Floer theory and gluing Donaldson polynomials, Topology 35 (1996), 167-200.

[6] H. U. Boden, Unitary representations of Brieskorn spheres, Duke Math. J. 75 (1994), 193-220.

[7] P. J. Braam and S. K. Donaldson, Floer's work on instanton homology, knots and surgery. In: The Floer memorial volume, vol. 133 of Progr. Math., Birkhäuser, Basel 1995, pp. $195-256$.

[8] P. J. Braam and S. K. Donaldson, Fukaya-Floer homology and gluing formulae for polynomial invariants In: The Floer memorial volume, vol. 133 of Progr. Math., Birkhäuser, Basel 1995, pp. 257-281.

[9] S. Cappell, R. Lee, and E. Miller, On the Maslov index, Comm. Pure Appl. Math. 47 (1994), 121-186.

[10] S. Cappell, R. Lee, and E. Miller, Self-adjoint elliptic operators and manifold decompositions. Part I: Low eigenmodes and stretching, Comm. Pure Appl. Math. 49 (1996), 825-866.

[11] S. Cappell, R. Lee, and E. Miller, Self-adjoint elliptic operators and manifold decompositions. Part II: Spectral flow and Maslov index, Comm. Pure Appl. Math. 49 (1996), 869-909.

[12] J. Conway, An enumeration of knots and links and some of their algebraic properties. In: Computational Problems in Abstract Algebra, J. Leech, ed., Pergamon Press, Oxford 1970, pp. 329-358.

[13] M. Daniel, A splitting formula for spectral flow. preprint, 1996.

[14] M. S. Farber and J. Levine, Jumps of the $\eta$-invariant, Math. Z. 223 (1996), 197-246. With an appendix by Shmuel Weinberger. 
[15] R. Fintushel and R. Stern, Pseudofree orbifolds, Annals of Math. 122 (1985), 335-364.

[16] R. Fintushel and R. Stern, Instanton homology of Seifert-fibered homology 3-spheres, Proc. Lond. Math. Soc. 61 (1990), 109-137.

[17] R. Fintushel and R. Stern, Integer graded instanton homology groups for homology threespheres, Topology 31 (1992), 589-604.

[18] A. Floer, An instanton invariant for 3-manifolds, Comm. Math. Phys. 118 (1989), 215-240.

[19] M. Furuta, $Z_{a}$-invariant $\mathrm{SU}(2)$ instantons over the four sphere. In: Geometry of lowdimensional manifolds, 1 (Durham, 1989), vol. 150 of London Math. Soc. Lecture Note Ser., Cambridge Univ. Press, Cambridge 1990, pp. 161-174.

[20] M. Furuta and Y. Hashimoto, Invariant instantons on $S^{4}$, J. Fac. Sci. Univ. Tokyo Sect. IA Math. 37 (1990), 585-600.

[21] R. Gompf and T. Mrowka, Irreducible 4-manifolds need not be complex, Annals of Math. 138 (1993), 61-111.

[22] C. Herald, Legendrian cobordism and Chern-Simons theory on 3-manifolds with boundary, Comm. Anal. Geom. 2 (1994), 337-413.

[23] J. Kania-Bartoszynska, Examples of different 3-manifolds with the same invariants of Witten and Reshetikhin-Turaev, Topology 32 (1993), 47-54.

[24] A. Kawauchi, Topological imitation, mutation and the quantum SU(2) invariant, J. Knot Theory Ramif. 3 (1994), 25-39.

[25] A. Kawauchi, Mutative hyperbolic homology 3-spheres with the same Floer homology, Geom. Dedicata 61 (1996), 205-217.

[26] R. Kirby, Problems in low-dimensional topology. In: Geometric Topology, W. Kazez, ed., American Math. Soc./International Press, Providence 1997.

[27] P. Kirk and E. Klassen, Computing spectral flow via cup products, J. Diff. Geo. 40 (1994), 505-562.

[28] P. A. Kirk, Mutations of homology spheres and Casson's invariant, Math. Proc. Camb. Phil. Soc. 105 (1989), 313-318.

[29] T. Kobayashi, Structures of the Haken manifolds with Heegaard splittings of genus two, Osaka J. Math. 21 (1984), 437-455.

[30] W. B. R. Lickorish, Distinct 3-manifolds with all SU(2) $)_{q}$ invariants the same, Proc. A.M.S. 117 (1993), 285-292.

[31] R. Meyerhoff and D. Ruberman, Cutting and pasting and the $\eta$-invariant, Duke J. Math. 61 (1990), 747-761.

[32] R. Meyerhoff and D. Ruberman, Mutation and the $\eta$-invariant, J. Diff. Geo. 31 (1990), 101-130.

[33] J. Morgan, T. Mrowka, and D. Ruberman, The $L^{2}$-moduli space and a vanishing theorem for Donaldson invariants, vol. 2 of Monographs in Geometry and Topology, International Press, 1994.

[34] T. Mrowka, P. Ozsváth, and B. Yu, Seiberg-Witten monopoles on Seifert fibered spaces, Comm. Anal. Geom. 5 (1997), 685-791.

[35] V. Muñoz, Donaldson invariants for connected sums along surfaces. Asian J. Math. 1 (1997), 785-800.

[36] L. I. Nicolaescu, The Maslov index, the spectral flow, and decompositions of manifolds, Duke Math. J. 80 (1995), 485-533.

[37] J. Porti, Torsion de Reidemeister pour les variétés hyperboliques., Mem. A.M.S. 128 (1997), $1-139$.

[38] P. Ramadevi, T. R. Govindarajan, and R. K. Kaul, Representations of composite braids and invariants for mutant knots and links in Chern-Simons field theories, Modern Phys. Lett. A 10 (1995), 1635-1658.

[39] Y. Rong, Mutation and Witten invariants, Topology 33 (1994), 499-507.

[40] D. Ruberman, Mutations and volumes of knots in $S^{3}$, Inventiones Math. 90 (1987), 189215. 
[41] C. Taubes, Casson's invariant and gauge theory, J. Diff. Geo. 31 (1990), 547-599.

Daniel Ruberman

Department of Mathematics

Brandeis University

Waltham, MA 02254

USA

e-mail: ruberman@binah.cc.brandeis.edu

(Received: November 20, 1998) 